\title{
Periodicity and Correlation Properties of $d$-FCSR Sequences
}

\author{
MARK GORESKY \\ School of Mathematics, Institute for Advanced Study, Princeton N.J. 08540 \\ goresky@ias.edu \\ ANDREW KLAPPER \\ klapper@cs.uky.edu \\ Department of Computer Science, 779 A Anderson Hall, University of Kentucky, Lexington, KY 40506-0046
}

Communicated by: P. Wild

Received September 17, 2001; Revised November 20, 2002; Accepted December 17, 2002

\begin{abstract}
A $d$-feedback-with-carry shift register ( $d$-FCSR) is a finite state machine, similar to a linear feedback shift register (LFSR), in which a small amount of memory and a delay (by $d$-clock cycles) is used in the feedback algorithm (see Goresky and Klapper [4,5]). The output sequences of these simple devices may be described using arithmetic in a ramified extension field of the rational numbers. In this paper we show how many of these sequences may also be described using simple integer arithmetic, and consequently how to find such sequences with large periods. We also analyze the "arithmetic crosscorrelation" between pairs of these sequences and show that it often vanishes identically.
\end{abstract}

Keywords: cross-correlations, 2-adic numbers, binary sequences, number field

AMS Classification: 94A55, 94A11, 11R04, 11S99

\section{Introduction}

Pseudorandom sequences have a wide array of uses in computer science and engineering including applications to spread spectrum communication systems, radar systems, signal synchronization, simulation, and cryptography. The pseudorandom sequences in a good family should (a) be easy to generate (possibly with hardware or software), (b) have good distribution properties which make them appear (statistically) to be "random", (c) have low crosscorrelation values so that each sequence may be separated from the others in the family, and (d) arise from some underlying algebraic structure so they can be analyzed using standard mathematical tools. (Of course other properties are desired as well, depending upon the application.) In this paper we continue the study begun in 1993 by the authors of a class of sequences based on the arithmetic of the $p$-adic numbers and their finite extensions [11] which enjoy all four of the above properties.

The simplest sequences of this type were described in terms of a class of efficient hardware generators called feedback with carry shift registers (FCSRs). An FCSR is structurally similar to a linear feedback shift register (LFSR), but whereas the analysis of LFSR sequences involves the algebra of power series with coefficients in 
$G F(2)$, the analysis of FCSR sequences involves the algebra of the $p$-adic numbers [14] (an elementary review of which is provided in Klapper and Goresky [11]). The analysis of FCSR sequences led to the cryptanalysis of the stream cipher known as the summation combiner [19]. It was further shown that various desirable statistical properties hold for FCSR sequences (see below), in most cases paralleling properties of LFSR sequences. FCSR sequences were discovered independently in a slightly different context by Marsaglia [17], and Marsaglia and Zaman [18] who showed they were useful as (pseudo-) random number generators for simulations. The simplest $p$-ary FCSR sequences may be described by

$$
a_{i}=A p^{-i}(\bmod N)(\bmod p) \in \mathbf{Z} /(p),
$$

where $p$ and $N$ are prime numbers for which $p$ is a primitive root (cf. Section 9) modulo $N$, where $A \in \mathbf{Z} /(N)$ and where $(\bmod N)(\bmod p)$ means that first the number $A p^{-i} \in \mathbf{Z} /(N)$ is represented by an integer between 0 and $N-1$; then this integer is reduced modulo $p$. (For most applications, $p$ will be 2.)

A wide variety of generalizations of FCSRs has been described. The case of interest in this paper, the $d$-FCSR, was described briefly in our papers [4] and [11]. It is the purpose of this paper to analyze these sequences. First we describe (Theorem 4.2) the output of a $d$-FCSR generator in terms of certain objects arising in algebraic number theory (namely, ramified extensions of the $p$-adic numbers). Then in Corollary 5.2 we show how these sequences can often (but not always) be described in terms of simple integer arithmetic:

$$
a_{i}=A b^{i}(\bmod N)(\bmod p),
$$

for values of $b \in \mathbf{Z} /(N)$ other than $b=p^{-1}$.

A much more general class of pseudorandom generators which includes LFSRs, FCSRs, and $d$-FCSRs all as special cases, was described by Klapper and Xu [13]. These generators are called algebraic feedback shift registers (AFSR). Many of the results in this paper are special cases of phenomena which occur for any AFSR, and some of these results were already described in Klapper and Xu [13]. The AFSR setting is the mathematically most natural setting in which to study shift register sequences. It also "explains" the many apparent similarities between the theory of LFSRs and the theory of FCSRs (and $d$-FCSRs). However in this paper we restrict our attention to the $d$-FCSRs. This paper is self-contained and does not rely on any results from Klapper and $\mathrm{Xu}[13]$.

In Goresky and Klapper [5] we show how to build shift register hardware which will generate these $d$-FCSR sequences with given design parameters. However, in this paper we maintain a purely algebraic point of view and we will only tangentially refer to the relevant shift register hardware. The constructions in Goresky and Klapper [5] depend heavily on this paper.

In the remaining sections our attention is restricted to the binary case, $p=2$. The usual notion of cross-correlation (with shift $\tau$ ) of two binary sequences $\mathbf{S}$ and $\mathbf{T}$ is the sum $\sum_{i}(-1)^{S_{i}+T_{i+\tau}}$. The addition in the exponent may be replaced by addition modulo 2 . Hence the cross-correlation with shift $\tau$ may be thought of as the number 
of ones minus the number of zeroes in one period of the sequence formed by adding $\mathbf{S}$ to the $\tau$-shift of $\mathbf{T}$, bit by bit modulo 2. Recently Klapper and Goresky [12] the authors considered a slightly different notion of cross-correlation between sequences: the arithmetic cross-correlation (with shift $\tau$ ) of $\mathbf{S}$ and $\mathbf{T}$ is the number of ones minus the number of zeroes in one period of (the periodic part of ) the sequence formed by adding $\mathbf{S}$ to the $\tau$-shift of $\mathbf{T}$ with carry. When $\mathbf{S}=\mathbf{T}$ the arithmetic cross-correlation is called the arithmetic autocorrelation. It was (to the best of our knowledge) first considered by Mandelbaum [16] who showed that certain binary sequences (the codewords of the Barrows-Mandelbaum arithmetic code) have "ideal" arithmetic autocorrelations: they are zero for every nontrivial shift $\tau$. The authors then showed [12] that certain families of FCSR sequences even have ideal arithmetic crosscorrelations. That is, all the nontrivial arithmetic cross-correlations are identically zero. The sizes of these families are conjectured to be huge, on the basis of experimental evidence. In Section 9 we further extend these results to $d$-FCSR sequences: we consider the $d$-arithmetic cross-correlation of two binary sequences and show that certain families of $d$-FCSR sequences have ideal $d$-arithmetic crosscorrelations. Results of this type contrast with the case of ordinary crosscorrelations, where there are well known lower bounds on the minimal cross-correlations in families of a given size. For example, the maximum shifted cross-correlation in a family of $M$ sequences of period $N$ is at least $M^{2} \sqrt{(M-1) /(M N-1)}[20]$.

The FCSR (and $d$-FCSR) sequences include the codewords of the BarrowsMandelbaum arithmetic codes as a special case. (We have previously referred to these as $\ell$-sequences, in order to stress the analogy with $m$-sequences.) Like the Barrows-Mandelbaum codes, since they have ideal arithmetic autocorrelations (respectively, $d$-arithmetic autocorrelations), they may be used for synchronization [16]. However since they also have ideal $(d-)$ arithmetic cross-correlations, our sequences may be used for simultaneous synchronization and identification in a multi-user environment.

To give a simple example, suppose a central dispatch $(D)$ wishes to send individual messages to one of a number of possible clients $\left(R_{1}, R_{2}, \ldots, R_{k}\right)$. Choose a family of (FCSR or $d$-FCSR) sequences with at least $k$ members; let $N$ denote the period of each sequence in this family. Each client $R_{i}$ is assigned a signature sequence $\mathbf{S}_{i}$ from the family. She monitors a common synchronization and identification channel on which $D$ broadcasts a bitstream $\mathbf{T}$. At the $n$-th clock tick, the client $R_{i}$ computes the $(d-)$ arithmetic cross-correlation $\Theta_{\mathbf{S}_{i}, \mathbf{T}}(n)$ between her signature sequence $\mathbf{S}_{i}$ and the current window of size $N$ in the bitstream $\mathbf{T}$.

If the dispatcher $D$ sends the $i$-th signature sequence $\mathbf{T}=\mathbf{S}_{i}$ on the common channel, the receiver $R_{i}$ will compute a single large correlation value $\Theta$ exactly $N$ clock ticks later, while the other clients will compute $\Theta_{\mathbf{S}_{j}}, \mathbf{T}(m)=0$ for all $j \neq i$ and for all shifts $m$. So the client $R_{i}$ has been identified as the intended recipient and her receiver has been synchronized to the message.

Finally, it was shown in Klapper and Goresky [11] that if the period of an FCSR sequence is maximal in a certain sense, then the distribution of subsequences of fixed length is nearly uniform (the numbers of occurrences of any two patterns of 
consecutive bits of fixed length differ by at most two). One expects these $d$-FCSR sequences to exhibit good distribution properties also; this question has been taken up in Klapper [10].

\section{Preliminaries}

Let $p \geq 2$ and $d \geq 1$ be integers such that the polynomial $X^{d}-p$ is irreducible over the rational numbers $\mathbf{Q}$. This occurs precisely when $p$ is not a $k$-th power, for any prime number $k$ dividing $d$ [15], p. 221. (In particular, it holds when $p$ is prime, which will be the main case of interest.) Let $\pi \in \mathbf{C}$ be a root of this polynomial. The set of all linear combinations $\sum_{i=0}^{d-1} a_{i} \pi^{i}$ with $a_{i} \in \mathbf{Q}$ is denoted $\mathbf{Q}[\pi]$. It is a field under the obvious operations of addition and multiplication (using $\pi^{d}=p$ ), and every nonzero element has an inverse (note that $\pi^{-1}=\frac{1}{p} \pi^{d-1}$ ) must use original. It is a degree $d$ (totally ramified) extension of the rational numbers $\mathbf{Q}$ and it is the smallest field extension of $\mathbf{Q}$ containing $\pi$. Having fixed $\pi \in \mathbf{C}$, we obtain an embedding $\mathbf{Q}[\pi] \subset \mathbf{C}$. However $\mathbf{Q}[\pi]$ actually admits $d$ different embeddings into the complex numbers, $\sigma_{i}: \mathbf{Q}[\pi] \rightarrow \mathbf{C}$ which are determined by setting $\sigma_{i}(\pi)=\zeta^{i} \pi$ (for $0 \leq i \leq d-1$ ) where $\zeta \in \mathbf{C}$ is a primitive $d$-th root of unity.

Similarly define $\mathbf{Z}[\pi]$ to be the ring consisting of all expressions

$$
u=\sum_{i=0}^{p-1} u_{i} \pi^{i}
$$

with $u_{i} \in \mathbf{Z}$. It is an entire ring in which every prime ideal is maximal. Every element of $\mathbf{Q}[\pi]$ may be expressed as a fraction $u / v$ with $u, v \in \mathbf{Z}[\pi]$. In other words, $\mathbf{Q}[\pi]$ is the fraction field of $\mathbf{Z}[\pi]$, and $\mathbf{Z}[\pi]$ is an "order" in $\mathbf{Q}[\pi]$ (although it does not necessarily coincide with the full ring of integers in $\mathbf{Q}[\pi]$, cf. Borevich and Shefarevich [2]).

Let $(\pi) \subset \mathbf{Z}[\pi]$ denote the ideal generated by $\pi$. The inclusion $\mathbf{Z} \rightarrow \mathbf{Z}[\pi]$ induces an isomorphism of rings $\mathbf{Z} /(p) \cong \mathbf{Z}[\pi] /(\pi)$. If $u \in \mathbf{Z}[\pi]$ is given by (3) then we denote by $\bar{u}$ its image in $\mathbf{Z}[\pi] /(\pi)$; it becomes identified with the number $u_{0}(\bmod p) \in \mathbf{Z} /(p)$ under this isomorphism. We shall often make (implicit) use of the vectorspace isomorphism

$$
\tau: \mathbf{Q}[\pi] \rightarrow \mathbf{Q}^{d},
$$

which is given by $\tau\left(\sum_{i=0}^{d-1} u_{i} \pi^{i}\right)=\left(u_{0}, u_{1}, \ldots, u_{d-1}\right)$. Then $\tau(\mathbf{Z}[\pi])=\mathbf{Z}^{d}$ which is a lattice in $\mathbf{Q}^{d}$.

The ring $\mathbf{Z}_{\pi}$ of $\pi$-adic integers consists of all (infinite) formal expressions $\alpha=$ $a_{0}+a_{1} \pi+a_{2} \pi^{2}+\cdots$ with $0 \leq a_{i} \leq p-1$, with the obvious operations of addition and multiplication (also using $\pi^{d}=p$ ). It is equal to the completion of the ring $\mathbf{Z}[\pi]$ at the ideal $(\pi)$ generated by $\pi$. If $d=1$ then $\pi=p, \mathbf{Z}[\pi]=\mathbf{Z}, \mathbf{Q}[\pi]=\mathbf{Q}$, and the $\pi$-adic integers are just the $p$-adic integers $\mathbf{Z}_{p}$. (See also Section 8.) 
The $\pi$-adic integers $\mathbf{Z}_{\pi}$ contains both $\mathbf{Z}_{p}$ (the $p$-adic integers) and $\mathbf{Z}[\pi]$ as well as many elements of $\mathbf{Q}[\pi]$. More, precisely, we have the following proposition, whose proof is just the same as that of Theorem 2.1 in Klapper and Goresky [11].

Proposition 2.1. The $\pi$-adic integer $\alpha=\sum_{i=0}^{\infty} a_{i} \pi^{i}$ (with $0 \leq a_{i} \leq p-1$ ) is in $\mathbf{Q}[\pi]$ if and only if its coefficient sequence $\left(a_{0}, a_{1}, a_{2}, \ldots\right)$ is eventually periodic. For any $u, q \in \mathbf{Z}[\pi]$, the fraction $u / q \in \mathbf{Q}[\pi]$ lies in $\mathbf{Z}_{\pi}$ if and only if the denominator $q=$ $\sum_{i=0}^{d-1} q_{i} \pi^{i}$ (with $q_{i} \in \mathbf{Z}$ ) satisfies either of the following three equivalent conditions,

- $q$ is invertible in $\mathbf{Z}_{\pi}$,

- the image of $q$ in $\mathbf{Z}[\pi] /(\pi)$ is invertible, or

- $q_{0}$ is relatively prime to $p$.

In this case, we say that $q$ is invertible $\bmod \pi$. The $\pi$-adic expansion

$$
\frac{u}{q}=\sum_{i=0}^{\infty} a_{i} \pi^{i} \in \mathbf{Z}_{\pi}
$$

(with $0 \leq a_{i} \leq p-1$ ) is then unique, and we refer to the (eventually periodic) sequence $a_{0}, a_{1}, \ldots$ as the coefficient sequence of $u / q$.

Definition 2.2. A $d$-FCSR sequence is the (eventually periodic) coefficient sequence of the $\pi$-adic expansion (5) of any fraction $u / q$, where $u, q \in \mathbf{Z}[\pi]$ and where $q$ is invertible $\bmod \pi$.

The denominator $q \in \mathbf{Z}[\pi]$ is called the connection number of the sequence. The purpose of this paper is to describe the (pseudorandom) sequences which are obtained this way (Theorem 4.2 and Corollary 5.2), and to investigate their correlation (Theorem 9.2) properties. It is a remarkable fact that (when $p=2$ ) such sequences may be generated using a simple shift register circuit whose feedback connections are determined by the choice of $q$ and whose initial loading is determined by the choice of $u$. These circuits were first described in Goresky and Klapper [4] and Klapper and Goresky [11] and are analyzed in Goresky and Klapper [5] using the results of this paper. The reader is referred to these other papers for applications and implementations of $d$-FCSR sequences.

\section{The Norm}

As in Section 2 suppose that $X^{d}-p$ is irreducible over $\mathbf{Q}$ and that $\pi \in \mathbf{C}$ is a root of this polynomial. In our analysis a central role is played by the algebraic norm of the connection number. The norm $N(x) \in \mathbf{Q}$ of an element $x=\sum_{i-0}^{d-1} x_{i} \pi^{i} \in \mathbf{Q}[\pi]$ is defined to be the determinant of the linear transformation $f_{x}(y)=x y$ on the field $\mathbf{Q}[\pi]$ (when it is considered as a $d$-dimensional vectorspace over $\mathbf{Q}$ ). With respect to 
the basis $\left\{1, \pi, \pi^{2}, \ldots, \pi^{d-1}\right\}$ the matrix of $f_{x}$ is given by

$$
\left(\begin{array}{ccccc}
x_{0} & p x_{d-1} & \ldots & p x_{2} & p x_{1} \\
x_{1} & x_{0} & \ldots & p x_{3} & p x_{2} \\
& & \ldots & & \\
x_{d-1} & x_{d-2} & \ldots & x_{1} & x_{0}
\end{array}\right) .
$$

The norm can also be computed as follows. Let $\zeta$ be a primitive $d$-th root of unity in the complex numbers and let $\sigma_{i}: \mathbf{Z}[\pi] \rightarrow \mathbf{C}$ be the above embedding defined by $\sigma_{i}(\pi)=\zeta^{i} \pi$. Then the norm of an element $x \in \mathbf{Q}[\pi]$ is the rational number

$$
N(x)=\prod_{i=0}^{d-1} \sigma_{i}(x) .
$$

If $x \in \mathbf{Z}[\pi]$, then $N(x) \in \mathbf{Z}$ is an integer.

LEMMA 3.1. Let $q \in \mathbf{Z}[\pi]$ and let $(q)$ denote the ideal in $\mathbf{Z}[\pi]$ generated by $q$. Then the absolute value $|N(q)|$ is equal to the number of elements in the quotient ring $\mathbf{Z}[\pi] /(q)$. The element $\delta=|N(q)| / q$ is in $\mathbf{Z}[\pi]$.

Proof. The proof of the first statement is standard and follows from Borevich and Shefarevich ([2], Lemma 1, p. 125): If $N \subset M$ are torsion free abelian groups of rank $n$ then the quotient $M / N$ is finite and has cardinality equal to the absolute value of the determinant of any transition matrix from a basis of $M$ to a basis of $N$. Take $M=\mathbf{Z}[\pi]$ with basis $\left\{1, \pi, \ldots, \pi^{d-1}\right\}$ and $N=(q)$ with basis $\left\{q, q \pi, \ldots, q \pi^{d-1}\right\}$. The transition matrix is that of the linear transformation $f_{q}(y)=q y$ whose determinant is also equal to the norm $N(q)$.

The second statement takes a bit more work. Let $q=\sum_{i=0}^{d-1} a_{i} \pi^{i}$ with $a_{i} \in \mathbf{Z}$. As in Section 2 , let $\zeta$ be a primitive $d$-th root of unity, so that by equation (7), we can write

$$
\delta= \pm \prod_{j=1}^{d-1} \sigma_{j}(q)= \pm \prod_{j=1}^{d-1} \sum_{i=0}^{d-1} a_{i} \zeta^{i j} \pi^{i}
$$

For any multi-index $I=\left(i_{1}, i_{2}, \ldots, i_{d-1}\right)$ (where $0 \leq i_{j} \leq d-1$ ), let $\mu(I)=E=$ $\left(e_{0}, e_{1}, \ldots, e_{d-1}\right)$ if for each $j$, the number of occurences of $j$ in the components of $I$ is exactly $e_{j}$. It follows that

$$
\delta= \pm \sum_{E}\left(\sum_{I: \mu(I)=E} \zeta^{\sum_{j=1}^{d-1} j i_{j}}\right) \prod_{i=0}^{d-1} a_{i}^{e_{i}} \pi^{i e_{i}},
$$

where the first sum is over all multi-indices $E=\left(e_{0}, e_{1}, \ldots, e_{d-1}\right)$ such that $0 \leq e_{j} \leq$ $d-1$ and $\sum e_{j}=d-1$. For each such multi-index $E$ let

$$
c_{E}(x)=\sum_{I: \mu(I)=E} x^{\sum_{j=1}^{d-1} j i_{j}} .
$$


Since the $a_{i}$ are integers, to prove the lemma it suffices to show that $c_{E}(\zeta)$ is an integer for each $E$. Note that $q$ has now left the picture.

The element $c_{E}(\zeta)$ lies in the field $\mathbf{Q}[\zeta]$, which is a Galois extension of the rational numbers. For an integer $k$, let $\tau_{k}$ be the homomorphism on $\mathbf{Q}[\zeta]$ induced by $\tau_{k}(\zeta)=\zeta^{k}$. The Galois group $\operatorname{Gal}(\mathbf{Q}[\zeta] / \mathbf{Q})$ consists of all $\tau_{k}$ such that $k$ is relatively prime to $d$ ([9], p. 195).

We claim that $c_{E}(\zeta)$ is a rational number. By Galois theory, to show this it suffices to show that $c_{E}\left(\tau_{k}(\zeta)\right)=c_{E}(\zeta)$ for every $\tau_{k}$ in the Galois group of $\mathbf{Q}[\zeta]$ over $\mathbf{Q}$. Let $k \ell \equiv 1(\bmod d)$. For any multi-index $I=\left(i_{1}, \ldots, i_{d-1}\right)$, let $i_{j}^{\prime}=i_{\ell j}$, and $I^{\prime}=$ $\left(i_{1}^{\prime}, \ldots, i_{d-1}^{\prime}\right)$ (here the index $\ell j$ in $i_{\ell j}$ is taken modulo $d$ ). The mapping from $I$ to $I^{\prime}$ is a permutation of the multi-indices $I$ with $\mu(I)=E$. Thus

$$
\begin{aligned}
c_{E}\left(\zeta^{k}\right) & =\sum_{I: \mu(I)=E} \zeta^{\sum_{j=1}^{d-1} k j i_{j}} \\
& =\sum_{I: \mu(I)=E} \zeta^{\sum_{j=1}^{d-1} j i_{l_{j}}} \\
& =\sum_{I: \mu\left(I^{\prime}\right)=E} \zeta^{\sum_{j=1}^{d-1} j i_{j}^{\prime}} \\
& =c_{E}(\zeta) .
\end{aligned}
$$

This proves the claim.

Furthermore, $c_{E}(\zeta)$ is integral over $\mathbf{Z}$ ( $\zeta$ is integral over $\mathbf{Z}$ since it is a root of the integral polynomial $x^{d}-1$. The sum and product of integral elements are integral). But the only elements of $\mathbf{Q}$ that are integral over $\mathbf{Z}$ are the integers. Thus $c_{E}(\zeta) \in \mathbf{Z}$, proving the lemma.

The second statement in Lemma 3.1 says that for any $q \in \mathbf{Z}[\pi]$ the norm $N(q)$ is divisible (in $\mathbf{Z}[\pi]$ ) by $q$. It follows that the composition $\mathbf{Z} \rightarrow \mathbf{Z}[\pi] \rightarrow \mathbf{Z}[\pi] /(q)$ induces a well-defined ring homomorphism

$$
\psi: \mathbf{Z} /(N(q)) \rightarrow \mathbf{Z}[\pi] /(q)
$$

Proposition 3.2. Suppose that $(q)=(r)^{t}$ for some $r \in \mathbf{Z}[\pi]$ and $t \in \mathbf{Z}^{+}$, and that $N(r)$ is (an ordinary, or "rational") prime. Then $(r) \subset \mathbf{Z}[\pi]$ is a prime ideal, $N(q)=N(r)^{t}$, and the mapping $\psi: \mathbf{Z} /(N(q)) \rightarrow \mathbf{Z}[\pi] /(q)$ is an isomorphism.

Proof. First consider the case $t=1$. The ring homomorphism $\psi$ is nontrivial since it takes 1 to 1 . So its image is a sub-ring of $\mathbf{Z} /(N(q))$ which is a field, so $\psi$ is surjective. Moreover the cardinality of $\mathbf{Z}[\pi] /(q)$ is $|N(q)|$ so $\psi$ is an isomorphism.

Now we proceed by induction on $t$. For any $s$ let us write $\psi=\psi_{s}: \mathbf{Z} /\left(N\left(r^{s}\right)\right) \rightarrow \mathbf{Z}[\pi] /\left(r^{s}\right)$. This induces a mapping between short exact 
sequences

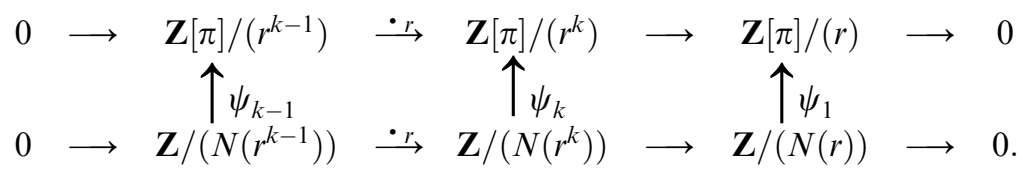

The horizontal maps are additive group homomorphisms giving short exact sequences. The vertical maps are ring homomorphisms. Since the vertical homomorphisms on the ends are isomorphisms by induction, the homomorphism in the middle must be a group isomorphism as well. But since it is also a ring homomorphism, it must be a ring isomorphism as well. Finally, the norm mapping is multiplicative so $N\left(r^{t}\right)=(N(r))^{t}$. This proves the proposition.

Proposition 3.2 says two things:

- If $u \in \mathbf{Z}[\pi]$ then there exists an integer $m \in \mathbf{Z}$ such that $m \equiv u(\bmod q)$. Moreover $m$ may be chosen so that $0 \leq m<|N(q)|$.

- If $a, b \in \mathbf{Z}$ are integers, then $a \equiv b(\bmod N(q))$ (as integers) if and only if $a \equiv b(\bmod q)($ as elements of $\mathbf{Z}[\pi])$.

\section{Periodicity}

As in Section 2 assume that $X^{d}-p$ is irreducible over $\mathbf{Q}$ and that $\pi \in \mathbf{C}$ is a root. In this section we identify the fractions $u / q \in \mathbf{Q}[\pi]$ whose $\pi$-adic expansions exist and are strictly periodic. Fix $q=\sum_{i=0}^{d-1} q_{i} \pi^{i} \in \mathbf{Z}[\pi]$ which is invertible in $\mathbf{Z}_{\pi}$. (By Proposition 2.1 this means that $q_{0}(\bmod p)$ is invertible in $\mathbf{Z} /(p)$.) Let $\delta=|N(q)| / q \in \mathbf{Z}[\pi]$.

LemmA 4.1. Let $u \in \mathbf{Z}[\pi]$ and set $\delta u=\sum_{i=0}^{d-1} z_{i} \pi^{i}$ with $z_{i} \in \mathbf{Z}$. Then the $\pi$-adic expansion of $u / q$ is obtained by interleaving the p-adic expansions of the $z_{i} /|N(q)|$. It is strictly periodic if and only if $-|N(q)| \leq z_{i} \leq 0$ for all $i(0 \leq i \leq d-1)$.

Proof. Calculating in $\mathbf{Q}[\pi]$,

$$
\frac{u}{q}=\frac{\delta u}{|N(q)|}=\sum_{i=0}^{d-1} \frac{z_{i}}{|N(q)|} \pi^{i} .
$$

So the $\pi$-adic expansion of $u / q$ is the interleaving of the $p$-adic expansions of the $z_{i} /|N(q)|$. Thus the former is strictly periodic if and only if each of the latter is strictly periodic. But the $p$-adic expansion of $z_{i} /|N(q)|$ is strictly periodic if and only if $-|N(q)| \leq z_{i} \leq 0$. (This fact is proven, for example, in Klapper and Goresky [11] for the case $p=2$ however the same proof works for general $p$.) 
Suppose that $q \in \mathbf{Z}[\pi]$ is invertible mod $\pi$ (cf. Proposition 2.1). Define the (half open) parallelepiped for $q$ to be

$$
P=\left\{\sum_{i=0}^{d-1} a_{i} q \pi^{i} \mid a_{i} \in \mathbf{Q} \text { and }-1<a_{i} \leq 0\right\} \subset \mathbf{Q}[\pi] .
$$

(The terminology refers to the fact that in equation (4) the image $\tau(P) \subset \mathbf{Q}^{d}$ is the parallelepiped spanned by the vectors $-q,-q \pi,-q \pi^{2}, \ldots,-q \pi^{d-1}$.) Define

$$
\Delta=P \cap \mathbf{Z}[\pi]
$$

to be the set of lattice points in this parallelepiped. Applying the projection to $\mathbf{Z}[\pi] /(q)$ gives a mapping

$$
\phi: \Delta \rightarrow \mathbf{Z}[\pi] /(q) .
$$

As in Goresky and Klapper [4], Klapper and Goresky [11], and Klapper and Xu [13] we have the following result.

THEOREM 4.2. If $q \in \mathbf{Z}[\pi]$ is invertible mod $\pi$ then the mapping $\phi$ is a one to one correspondence. For any element $u \in \Delta$, the $\pi$-adic expansion

$$
\frac{u}{q}=\sum_{i=0}^{\infty} a_{i} \pi^{i}
$$

of the fraction $u / q$ is strictly periodic with

$$
a_{i}=\bar{q}^{-1}\left(\pi^{-i} u(\bmod q)\right)(\bmod \pi) .
$$

This last equation needs a bit of explanation. Using the fact that $\phi$ is a one to one correspondence, for any element $x \in \mathbf{Z}[\pi] /(q)$ define

$$
x(\bmod \pi)=\phi^{-1}(x)(\bmod \pi) \in \mathbf{Z}[\pi] /(\pi)=\mathbf{Z} /(p),
$$

meaning that we first lift $x$ up to $\Delta$ and then reduce modulo $\pi$. The image of $\pi$ in $\mathbf{Z}[\pi] /(q)$ is invertible, so the product $x=\pi^{-i} u$ is defined in $\mathbf{Z}[\pi] /(q)$. Hence

$$
\pi^{-i} u(\bmod \pi)=\phi^{-1}\left(\pi^{-i} u(\bmod q)\right)(\bmod \pi)
$$

is defined in $\mathbf{Z} /(p)$. Moreover, the element

$$
\bar{q}=q(\bmod \pi) \in \mathbf{Z}[\pi] /(\pi)=\mathbf{Z} /(p)
$$

is also invertible; by (6) it coincides with $q_{0}(\bmod p)$. So the product (11) makes sense in $\mathbf{Z} /(p)$. We remark that the mapping (12), $\mathbf{Z}[\pi] /(q) \rightarrow \mathbf{Z} /(p)$ is not a ring homomorphism and in fact it depends on the choice $\Delta \subset \mathbf{Z}[\pi]$ of a complete set of representatives for the elements of $\mathbf{Z}[\pi] /(q)$. 
Proof. We need to show that the composition $\Delta \subset \mathbf{Z}[\pi] \rightarrow \mathbf{Z}[\pi] /(q)$ is one to one and surjective. The idea is that existence and uniqueness of representatives $(\bmod q)$ in $P$ corresponds to existence and uniqueness $(\bmod |N(q)|)$ in the (half-open) hypercube

$$
\delta P=\left\{\sum_{i=0}^{d-1} a_{i} \pi^{i} \mid a_{i} \in \mathbf{Q} \text { and }-|N(q)|<a_{i} \leq 0\right\},
$$

(the image of $P$ under the linear map $\mathbf{Q}[\pi] \rightarrow \mathbf{Q}[\pi]$ which is given by multiplication by $\delta)$. First we verify surjectivity. For any $z \in \mathbf{Q}$ define $\hat{z} \in \mathbf{Q}$ to be the unique rational number such that $-|N(q)|<\hat{z} \leq 0$ and such that $z-\hat{z}=m|N(q)|$ for some integer $m$. If $z$ is an integer then so is $\hat{z}$.

Now let $x \in \mathbf{Z}[\pi]$ be arbitrary, and write $\delta x=\sum_{i=0}^{d-1} x_{i} \pi^{i}$ for some integers $x_{i}$. Set $y=\sum_{i=0}^{d-1} \hat{x}_{i} \pi^{i}$. We claim that $\delta^{-1} y \in \Delta$ and that it is congruent to $x$ modulo $q$. In fact,

$$
\begin{aligned}
x-\delta^{-1} y & =\delta^{-1}(\delta x-y) \\
& =\delta^{-1} \sum_{i=0}^{d-1}\left(x_{i}-\hat{x}_{i}\right) \pi^{i} \\
& =\delta^{-1} \sum_{i=0}^{d-1} m_{i}|N(q)| \pi^{i} \\
& =q \sum_{i=0}^{d-1} m_{i} \pi^{i}
\end{aligned}
$$

(for some integers $m_{i}$ ). This last expression is in $\mathbf{Z}[\pi]$, hence $\delta^{-1} y \in \mathbf{Z}[\pi]$. But $\delta^{-1} y$ is also in $P$, so $\delta^{-1} y \in \Delta$ and (again by the last expression) it is congruent to $x$ modulo $(q)$. To prove injectivity, suppose that $x, x^{\prime} \in \Delta$ are congruent modulo $(q)$. Then $x-x^{\prime}=c q$ for some $c \in \mathbf{Z}[\pi]$, so $\delta x-\delta x^{\prime}=c \delta q=c|N(q)|$. But both $\delta x$ and $\delta x^{\prime}$ lie in the half-open hypercube $\delta P$ whose sides all have length $|N(q)|$. So no coordinate can differ by as much as $|N(q)|$, hence $c=0$. The statement about strictly periodic $\pi$-adic expansions follows immediately from Lemma 4.1.

Now let us verify (11). The sequence $\left\{a_{1}, a_{2}, \ldots\right\}$ is also strictly periodic and corresponds to a $\pi$-adic integer $u^{\prime} / q=\sum_{i=0}^{\infty} a_{i+1} \pi^{i}$ with the same denominator $q$ (and with some numerator $u^{\prime} \in \Delta$ ). Then

$$
\frac{u^{\prime}}{q} \pi=\frac{u}{q}-a_{0} \quad \text { or } \quad u^{\prime} \pi=u-a_{0} q \in \mathbf{Z}[\pi] .
$$

Reducing this equation mod $\pi$ gives the first case of (11), $a_{0} \equiv \bar{q}^{-1} u(\bmod \pi)$. (Since the element $u$ is already in $\Delta$ it is not necessary to "lift" it.) Similarly,

$$
a_{1} \equiv \bar{q}^{-1} u^{\prime}(\bmod \pi) .
$$

Reducing (13) modulo $q$ gives $u^{\prime} \equiv \pi^{-1} u(\bmod q) \in \mathbf{Z}[\pi] /(q)$. Since $\phi: \Delta \rightarrow \mathbf{Z}[\pi] /(q)$ is a bijection, this element $u^{\prime}$ is the unique "lift" of the quantity $\pi^{-1} u(\bmod q) \in \mathbf{Z}[\pi] /(q)$. 
Substituting into (14) gives

$$
a_{1}=\bar{q}^{-1}\left(\pi^{-1} u\right)(\bmod q)(\bmod \pi)
$$

where the operation $z \mapsto z(\bmod q)(\bmod \pi)$ means that first $z \in \mathbf{Z}[\pi] /(q)$ is lifted to $\Delta \subset \mathbf{Z}[\pi]$ then reduced modulo $\pi$. Now equation (11) follows by induction.

Remark. A face $F \subset P$ of the parallelepiped (8) is the set of points obtained by setting some of the coefficients $a_{j}=0$. The set of lattice points $\Delta \cap F$ in any face correspond under equation (9) to an additive subgroup of $\mathbf{Z}[\pi] /(q)$. If $N(q)$ is prime then there are no additive subgroups other than $\{0\}$. Hence, if $N(q)$ is prime, all the nonzero elements of $\Delta$ lie in the interior of the parallelepiped.

In order to reconcile the notation with that of Goresky and Klapper [5] we briefly consider the special case $p=2$. Then $\mathbf{Z}[\pi] /(\pi)=\mathbf{Z} /(2)$. If $q \in \mathbf{Z}[\pi]$ is invertible mod $\pi$ (meaning that $q_{0}$ is odd) then $\bar{q}^{-1}=1$. Let

$$
P^{\prime}=\left\{\sum a_{i} q \pi^{i} \in \mathbf{Z}[\pi] \mid a_{i} \in \mathbf{Q} \text { and } 0 \leq a_{i}<1\right\}
$$

and set $\Delta^{\prime}=P^{\prime} \cap \mathbf{Z}[\pi]$. Then $\Delta^{\prime}$ is the "negative" of $\Delta$ and, like $\Delta$ it is a complete set of representatives for the elements of $\mathbf{Z}[\pi] /(q)$, that is, the mapping

$$
\phi^{\prime}: \Delta^{\prime} \rightarrow \mathbf{Z}[\pi] /(q)
$$

(which is given by reduction modulo $q$ ) is a one-to-one correspondence. Fix $u \in \Delta$ and set $h=-u \in \Delta^{\prime}$. Then Theorem 4.2 becomes:

COROLlary 4.3. Suppose that $d=2$, that $q$ is invertible $\bmod \pi$, and that $h \in \Delta^{\prime}$. Then the $\pi$-adic expansion

$$
\frac{-h}{q}=\sum_{i=0}^{\infty} b_{i} \pi^{i}
$$

of the fraction $-h / q$ is strictly periodic with

$$
b_{i}=\pi^{-i} h(\bmod q)(\bmod \pi)
$$

where the operation $z(\bmod q)(\bmod \pi)$ means that first the element $z \in \mathbf{Z}[\pi] /(q)$ is lifted to the complete set of representatives $\Delta^{\prime}$, and then reduced modulo $q$.

We now return to the general case. The following lemma will be used in the proof of Proposition 9.3 to analyze arithmetic correlations. 
Lemma 4.4. Suppose $x \in \Delta$. Then the representative of $-x$ modulo $q$ in $\Delta$ is

$$
y=-q \frac{\pi^{d}-1}{\pi-1}-x .
$$

Proof. The element $x$ is in $\Delta$ if and only if each $\pi$-adic coordinate $z_{i}$ of $\delta x$ is between $-|N(q)|$ and 0 . If this holds, then

$$
-|N(q)|<-|N(q)|-z_{i}<0 .
$$

But $-|N(q)|-z_{i}$ is the $i$-th $\pi$-adic coordinate of $-|N(q)|\left(1+\pi+\pi^{2}+\cdots+\right.$ $\left.\pi^{d-1}\right)-\delta x=\delta y$. Thus $y$ is in $\Delta$ and is congruent to $-x$ modulo $q$.

\section{Elementary Description of $d$-FCSR Sequences}

In most cases it is possible to describe the $d$-FCSR sequences in elementary terms (without reference to algebraic number fields). This is the first main result of the paper.

As in Sections 2 and 3, suppose that $\pi^{d}=p$ and that $X^{d}-p$ is irreducible over $\mathbf{Q}$. Let $q \in \mathbf{Z}[\pi]$ and suppose that

- $q$ is invertible in $\mathbf{Z}_{\pi}$,

- $d$ is relatively prime to $N(q)$,

- $(q)=\left(r^{t}\right)$ for some $r \in \mathbf{Z}[\pi]$, and

- $N(r)$ is (a "rational", or ordinary) prime.

Recall that all these conditions are satisfied if $p$ is prime and $N(q)$ is prime.

Let $N$ denote $|N(q)|$. By Proposition 3.2 we have a natural isomorphism of rings, $\psi: \mathbf{Z} /(N) \rightarrow \mathbf{Z}[\pi] /(q)$. Each of these rings has a canonical set of representatives: let

$$
S=\{0,-1,-2, \ldots,-(N-1)\} \subset \mathbf{Z},
$$

and let $\Delta \subset \mathbf{Z}[\pi]$ be the parallelepiped as defined as in Section 4. Then $S$ is a complete set of representatives for the elements of $\mathbf{Z} /(N)$ and $\Delta$ is a complete set of representatives for the elements of $\mathbf{Z}[\pi] /(q)$. By abuse of notation we shall also write $\psi: S \rightarrow \Delta$ for the resulting one-to-one correspondence. By Lemma 3.1 the element $\delta=|N(q)| / q$ is in $\mathbf{Z}[\pi]$ and may be expressed as $\delta=\sum_{i=0}^{d-1} s_{i} \pi^{i}$.

THEOREM 5.1. Let $u \in \Delta$. Then in $\mathbf{Z} /(p)$,

$$
\left(\bar{q}^{-1} u\right)(\bmod \pi)=\bar{N}(q)^{-1}\left(s_{0} \psi^{-1}(u)(\bmod N)\right)(\bmod p) .
$$

The left side of this equation makes sense since the image $\bar{q} \in \mathbf{Z}[\pi] /(\pi)$ of $q \in \mathbf{Z}[\pi]$ is invertible. On the right side of the equation, the element $\psi^{-1}(u) \in \mathbf{Z} /(N)$ is multiplied by $s_{0}(\bmod N)$ then lifted to the set $S$ of representatives. Then the result is reduced 
modulo $p$. The image $\bar{N}(q) \in \mathbf{Z} /(p)$ of $N(q) \in \mathbf{Z}$ is invertible, so the product on the right hand side makes sense. This result may be best explained in terms of the following two diagrams. Both the upper and lower squares in the first diagram commute. Although the upper square in the second diagram commutes, the lower square does not. Theorem 5.1 supplies "correction factors" which are needed in order to make it commute.
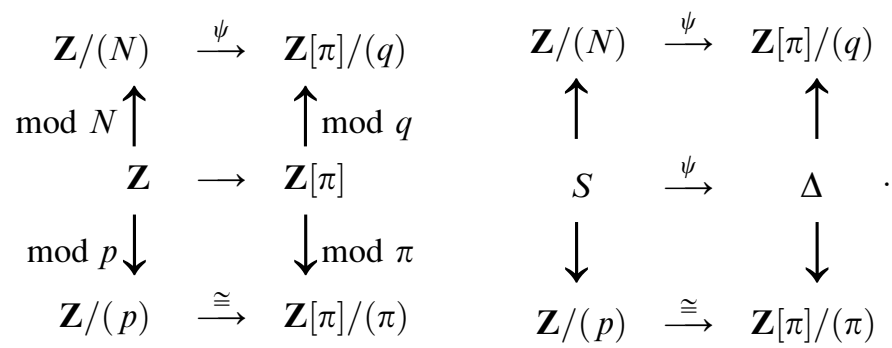

Replacing $u$ with $\bar{\pi}^{-i} u$ in Theorem 5.1 gives the following corollary which describes the $d$-FCSR sequence completely in terms of operations on ordinary integers.

Corollary 5.2. Let $u \in \Delta$ and let $m=\psi^{-1}(\pi) \in \mathbf{Z} /(N)$. Write $u / q=\sum_{j=0}^{\infty} a_{j} \pi^{j}$ for the $\pi$-adic expansion of the fraction $u / q$. Then the coefficient sequence $a_{0}, a_{1}, \ldots$ is strictly periodic and moreover

$$
a_{j}=\bar{N}(q)^{-1}\left(A b^{i}(\bmod N)\right)(\bmod p),
$$

where $b=m^{-1}$ and $A=s_{0} \psi^{-1}(u) \in \mathbf{Z} /(N)$.

The proof of Theorem 5.1 will occupy the next section.

\section{Proof of Theorem 5.1}

Continue with the same notation as in Section 4, that is, $\pi^{d}=p, q \in \mathbf{Z}[\pi]$, $N=|N(q)|, \quad \delta=N(q) / q=\sum_{i=0}^{d-1} s_{i} \pi^{i}$ and $m=\psi^{-1}(\pi) \in \mathbf{Z} /(N)$. Let $\zeta \in \mathbf{C}$ be a primitive $d$-th root of unity and let $R$ denote any one of the following rings: $\mathbf{Z}, \mathbf{Z}[\pi], \mathbf{Z}[\zeta], \mathbf{Z}[\pi, \zeta]$. The integer $N \in \mathbf{Z}$ generates an ideal $(N)_{R}$ in the ring $R$. If $a, b \in \mathbf{Z}$ we write

$$
a \equiv b(\bmod N) \text { in } R,
$$

to mean that $a-b \in(N)_{R}$. 
LEMMA 6.1. For any $a, b \in \mathbf{Z}$ we have

$a \equiv b(\bmod N)$ in $R \Longleftrightarrow a \equiv b(\bmod N)$ in $\mathbf{Z}$.

Proof. Both $\pi$ and $\zeta$ are integral over $\mathbf{Z}$ so each $x \in R$ is integral over $\mathbf{Z}$. If $a \equiv b(\bmod N)$ in $R$, then $a-b=x N$ for some $x \in R$. Then $x=(a-b) / N$ is a rational number which is integral over $\mathbf{Z}$, hence $x \in \mathbf{Z}$.

LEMMA 6.2. The inclusion $\mathbf{Z}[\zeta] \rightarrow \mathbf{Z}[\pi, \zeta]$ induces a ring isomorphism

$$
\Psi: \mathbf{Z}[\zeta] /(N) \cong \mathbf{Z}[\pi, \zeta] /(q)
$$

Proof. The proof is similar to that of Proposition 3.2. Since $q \mid N$ in $\mathbf{Z}[\pi, \zeta]$ the mapping $\Psi$ is well defined. To see it is surjective it suffices to show that $\pi$ is in the image of $\phi$. But $\pi \equiv m(\bmod q)$ in $\mathbf{Z}[\pi]$, hence also in $\mathbf{Z}[\pi, \zeta]$, that is, $\Psi(m)=\pi$. Finally, the cardinality of both rings is $|N|^{\phi(d)}$ (where $\phi$ is the Euler totient function), hence $\Psi$ is also injective.

LEMMA 6.3. Let $u \in \mathbf{Z}[\pi]$ and set $\delta u=\sum_{i=0}^{d} z_{i} \pi^{i}$. Then $z_{k} \pi^{k} \equiv z_{0}(\bmod q)($ in $\mathbf{Z}[\pi])$ and $z_{k} m^{k} \equiv z_{0}(\bmod N)($ in $\mathbf{Z})$ for all $k=0,1, \ldots, d$.

Proof. Let $\hat{z}_{j}$ denote the $j$-th Fourier coefficient of the finite sequence of complex numbers

$$
\left(z_{0}, z_{1} \pi, z_{2} \pi^{2}, \ldots, z_{d-1} \pi^{d-1}\right)
$$

which may be considered to be a function $f: \mathbf{Z} /(d) \rightarrow \mathbf{C}$. In other words, if $\zeta \in \mathbf{C}$ denotes a primitive $d$-th root of unity, then

$$
\hat{z}_{j}=\sum_{i=0}^{d-1} z_{i} \pi^{i \zeta^{i j}}
$$

Evidently, the Fourier coefficient $\hat{z}_{j}$ lies in the ring $\mathbf{Z}[\pi, \zeta]$. The homomorphism $\sigma_{j}: \mathbf{Z}[\pi] \rightarrow \mathbf{C}$ may be extended to this ring by setting $\sigma_{j}(\zeta)=\zeta$. Then

$$
\sigma_{j}(\delta)=\prod_{i \neq j} \sigma_{i}(q)
$$

which implies that, in the ring $\mathbf{Z}[\pi, \zeta]$, the element $\sigma_{j}(\delta)$ is divisible by $q$, for 
$j=1,2, \ldots, d-1$. Hence

$$
\begin{aligned}
\hat{z}_{j} & =\sum_{i=0}^{d-1} z_{i} \pi^{i} \zeta^{i j} \\
& =\sigma_{j}(\delta u) \\
& =\sigma_{j}(\delta) \sigma_{j}(u) \\
& \equiv 0(\bmod q) .
\end{aligned}
$$

The Fourier inversion formula gives

$$
\begin{aligned}
d z_{k} \pi^{k} & =\sum_{j=0}^{d-1} \hat{z}_{j} \zeta^{-k j} \\
& \equiv \hat{z}_{0}(\bmod q)
\end{aligned}
$$

which is independent of $k$. Since $d$ is invertible $\bmod N(q)$ it is also invertible in $\mathbf{Z}[\pi] /(q)$, meaning that $d x=1+y q$ for some $x, y \in \mathbf{Z}[\pi]$. Hence $d$ is also invertible in $\mathbf{Z}[\pi, \zeta] /(q)$, which shows that

$$
z_{k} \pi^{k} \equiv z_{0} \pi^{0}(\bmod q) \text { in } \mathbf{Z}[\pi, \zeta]
$$

for all $k$. We need to show the same holds in $\mathbf{Z}[\pi]$. However,

$$
\begin{aligned}
z_{k} \pi^{k} & \equiv z_{0}(\bmod q) \text { in } \mathbf{Z}[\pi, \zeta] \\
\Rightarrow z_{k} m^{k} & \equiv z_{0}(\bmod q) \text { in } \mathbf{Z}[\pi, \zeta] \\
\Rightarrow z_{k} m^{k} & \equiv z_{0}(\bmod N) \text { in } \mathbf{Z}[\zeta] \\
\Rightarrow z_{k} m^{k} & \equiv z_{0}(\bmod N) \text { in } \mathbf{Z} \\
\Rightarrow z_{k} m^{k} & \equiv z_{0}(\bmod q) \text { in } \mathbf{Z}[\pi] \\
\Rightarrow z_{k} \pi^{k} & \equiv z_{0}(\bmod q) \text { in } \mathbf{Z}[\pi],
\end{aligned}
$$

by Lemmas (6.1), (6.2) and Proposition (3.2).

\subsection{Proof of Theorem 5.1}

As in the previous section set $\delta u=\sum_{k=0}^{d-1} z_{k} \pi^{k}$ and set $\delta=\sum_{k=0}^{d-1} s_{k} \pi^{k}$. Lemma 6.3 gives $\delta u \equiv d z_{0}(\bmod q)$ and $\delta \equiv d s_{0}(\bmod q)$, hence $d z_{0} \equiv d s_{0} u(\bmod q)$. Since $d$ is invertible $(\bmod q)$, this implies $z_{0} \equiv s_{0} u(\bmod q)$. The isomorphism $\psi^{-1}$ : 
$\mathbf{Z}[\pi] /(q) \rightarrow \mathbf{Z} /(N)$ is the identity on integers, so we obtain

$$
z_{0}=s_{0} \psi^{-1}(u) \text { in } \mathbf{Z} /(N) .
$$

Now lift these elements to the set $S \subset \mathbf{Z}$ of representatives of $\mathbf{Z} /(N)$ and reduce modulo $p$ to obtain

$$
z_{0}(\bmod p)=\left(s_{0} \psi^{-1}(u)(\bmod N)\right)(\bmod p) .
$$

But $z_{0}(\bmod p)=\delta u(\bmod \pi) \in \mathbf{Z} /(p)$. That is,

$$
(\delta u)(\bmod \pi)=\left(s_{0} \psi^{-1}(u)(\bmod N)\right)(\bmod p) .
$$

Multiplying by $\bar{N}(q)^{-1} \in \mathbf{Z} /(p)$ gives

$$
\left(\bar{q}^{-1} u\right)(\bmod \pi)=\bar{N}(q)^{-1}\left(s_{0} \psi^{-1}(u)(\bmod N)\right)(\bmod p),
$$

where $\bar{q} \in \mathbf{Z}[\pi] /(\pi)$ is the image of $q$, and where the right hand side is interpreted as follows: first compute $s_{0} \psi^{-1}(u) \in \mathbf{Z} /(N)$, then lift this to the set $S$ of representatives, then reduce modulo $p$, then multiply by $\bar{N}(q)^{-1}$. This completes the proof of Theorem 5.1.

\section{Example}

Consider a binary $d$-FCSR (meaning that $p=2$ ) with $\pi^{2}=2$ (so $d=2$ ) and with connection number $q \in \mathbf{Z}[\pi]$ which is invertible in $\mathbf{Z}_{\pi}$. Let $N=|N(q)|$. Then $\bar{N}(q)^{-1}=1$ and Corollary 5.2 says that the strictly periodic portions of the $d$-FCSR sequence will be described by

$$
a_{j}=A b^{j}(\bmod N)(\bmod 2),
$$

for certain $A, b \in \mathbf{Z} /(N)$. Let us take $q=5+2 \pi$. Then $N(q)=17$ which is prime, so the parallelogram contains 16 elements in its interior. It is illustrated in Figure 1.

The isomorphism $\psi: \mathbf{Z}[\pi] /(q) \rightarrow \mathbf{Z} /(17)$ maps $\pi$ to $m=6$. Hence $m^{-1}=3 \in \mathbf{Z} /(17)$ which is primitive, so we obtain a maximal length output sequence. The element $\delta=5-2 \pi$ hence $s_{0}=5$. Each element in $\mathbf{Z}[\pi] /(q)$ has a unique representative $u$ in the parallelogram; these representatives are listed in the second column of the table in Figure 2. Each element in $\mathbf{Z} /(17)$ has a unique representative in the set $S=\{-1,-2, \ldots,-16\}$; these representatives $h$ are listed in the third column. The correspondence between the second and third column is given by Theorem 5.1, that is, $h=s_{0} \psi^{-1}(u)$ (since $\bar{q} \equiv \bar{N}(q) \equiv 1(\bmod 2)$ ). The fourth column (which is the $d$ FCSR sequence under consideration) is the third column modulo 2 , and it concides with the second column modulo $\pi$. 


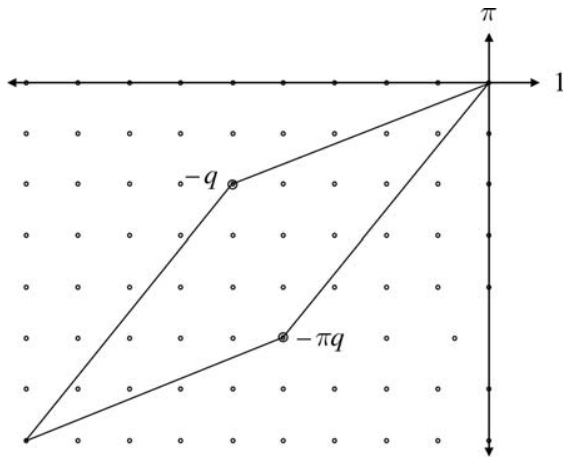

Figure 1. Parallelogram for $q=5+2 \pi$.

\begin{tabular}{|c|c|c|c|}
\hline$i$ & $\pi^{-i}(\bmod q)$ & $5 \cdot 6^{-i}(\bmod 17)$ & output \\
\hline \hline 0 & $-4-2 \pi$ & -12 & 0 \\
1 & $-2-2 \pi$ & -2 & 0 \\
2 & $-2-\pi$ & -6 & 0 \\
3 & $-1-\pi$ & -1 & 1 \\
4 & $-3-3 \pi$ & -3 & 1 \\
5 & $-5-4 \pi$ & -9 & 1 \\
6 & $-6-5 \pi$ & -10 & 0 \\
7 & $-5-3 \pi$ & -13 & 1 \\
8 & $-5-5 \pi$ & -5 & 1 \\
9 & $-7-5 \pi$ & -15 & 1 \\
10 & $-7-6 \pi$ & -11 & 1 \\
11 & $-8-6 \pi$ & -16 & 0 \\
12 & $-6-4 \pi$ & -14 & 0 \\
13 & $-4-3 \pi$ & -8 & 0 \\
14 & $-3-2 \pi$ & -7 & 1 \\
15 & $-4-4 \pi$ & -4 & 0 \\
\hline
\end{tabular}

Figure 2. Model states for $q=5+2 \pi$.

\section{8. $\quad$ Arithmetic in $Z_{\pi}$}

The comments in this section will be needed for Section 9. As in Section 2, let $\pi^{d}=p$. A $p$-ary sequence $\mathbf{a}=a_{0}, a_{1}, \ldots$ is an infinite sequence of symbols with $0 \leq a_{i} \leq p-1$. Such a sequence is the coefficient sequence of a unique $\pi$-adic integer

$$
\alpha=\sum_{i=0}^{\infty} a_{i} \pi^{i} \in \mathbf{Z}_{\pi} .
$$

Addition of $\pi$-adic integers $\alpha=\sum_{i=0}^{\infty} a_{i} \pi^{i}$ and $\beta=\sum_{i=0}^{\infty} b_{i} \pi^{i}$ is accomplished by adding their coefficients symbol by symbol, using a "carry" operation which delays each carried bit $d$ steps before adding it back in. In other words, if $\alpha+\beta=\sum_{i=0}^{\infty} e_{i} \pi^{i}$ 
with $0 \leq e_{i} \leq p-1$ then

$$
a_{i}+b_{i}+c_{i}=e_{i}+p c_{i+d},
$$

where $c_{i}$ denotes the amount carried to the $i$-th position. Since $c_{0}=0$, it follows immediately that $c_{i}$ is either 0 or 1 . Similarly the difference $\alpha-\beta=\sum_{i=0}^{\infty} f_{i} \pi^{i}$ is obtained by subtracting the coefficients symbol by symbol, using a "borrow" operation which is delayed $d$ steps. The "borrow" operation is actually the same as the "carry" operation, but the carried quantity is negative, that is,

$$
a_{i}-b_{i}+c_{i}=f_{i}+p c_{i+d},
$$

from which it also follows immediately that the amount $c_{i}$ to be carried to the $i$-th place is either 0 or -1 .

EXAMPLE. If the coefficient sequences of $\alpha, \beta \in \mathbf{Z}_{\pi}$ are eventually periodic of the same period, say $\mathrm{L}$, the coefficient sequence of $\alpha \pm \beta$ may have a larger period. In the following example, $p=2$ and $d=4$. In the table the "carries" are indicated in the row c. Spaces are provided to help distinguish the periods of $\alpha$ and $\beta$, which are periodic with period $L=6$. We see that the coefficient sequence for $\gamma=\alpha+\beta$ is eventually periodic with period 12. Although the periodic part of $\alpha$ and $\beta$ begins at $i=2$ the periodic part of $\gamma$ does not begin until $i=14$.

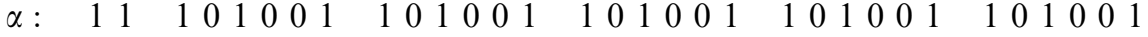

$$
\begin{aligned}
& \beta: \quad 110010100001010000101000101010001010100 \\
& c: \quad \quad \begin{array}{llllllllll}
11 & 11 & 11 & 1 & 1 & 1 & 1
\end{array} \\
& \gamma: \quad 00 \quad 110001 \quad 001110 \quad 111001 \quad 101100 \quad 111001 .
\end{aligned}
$$

LEMMA 8.1. Let $\alpha=\sum_{i=0}^{\infty} a_{i} \pi^{i}$ and $\beta=\sum_{i=0}^{\infty} b_{i} \pi^{i}$ be $\pi$-adic integers whose coefficient sequences are eventually periodic with period dividing $L$. Then the coefficient sequence of $\alpha \pm \beta$ is eventually periodic with period dividing $\operatorname{lcm}(L, d)$. If the coefficient sequences of $\alpha$ and $\beta$ are strictly periodic beyond index $k$, then the coefficient sequence of $\alpha \pm \beta$ is strictly periodic beyond index $k+\operatorname{lcm}(L, d)$. Moreover, if $\alpha$ and $\beta$ are strictly periodic, then the eventual period is a divisor of $L$.

Proof. We only consider the case of $\alpha+\beta$; the case of the difference is similar. Suppose that $\alpha$ and $\beta$ are strictly periodic beyond index $k$. First consider the case $d=1$, so $\pi=p$. In this case we have $\alpha=\alpha_{0}+p^{k} \alpha_{1}$ and $\beta=\beta_{0}+p^{k} \beta_{1}$ with $0 \leq \alpha_{0}, \beta_{0}<p^{k}$, where the coefficient sequences of $\alpha_{1}$ and $\beta_{1}$ are strictly periodic. It follows (cf. Lemma 4.1) that $\alpha_{1}=a^{\prime} /\left(p^{L}-1\right)$ and $\beta_{1}=b^{\prime} /\left(p^{L}-1\right)$ for some integers $-p^{L}+1 \leq a^{\prime}, b^{\prime} \leq 0$. Also, $\alpha_{0}+\beta_{0}=\delta_{0}+c p^{k}$ with $0 \leq \delta<p^{k}$ and $c \in\{0,1\}$. Therefore for some $e$ with $-p^{L}+1<e \leq 0, d \in\{0,-1\}$, and $\gamma$ with strictly periodic coefficient sequence, we have

$$
\alpha_{1}+\beta_{1}=\frac{a^{\prime}+b^{\prime}}{p^{L}-1}=d+\frac{e}{p^{L}-1}=d+\gamma .
$$


Thus

$$
\alpha+\beta=\delta_{0}+p^{k}(c+d+\gamma),
$$

with $c+d \in\{-1,0,1\}$. If $c+d=0$, the conclusion of the lemma holds.

Suppose $c+d=1$. If every coefficient of $\gamma$ is $p-1$, then $c+d+\gamma=0$, which is strictly periodic. Otherwise at least one coefficient is less than $p-1$, so there is no carry beyond this place when we add $c+d+\gamma$. Since $\gamma$ has period $L, \alpha+\beta$ has the same eventual period and its periodic part starts by index $k+L$.

Suppose $c+d=-1$. If every coefficient of $\gamma$ is 0 , then $c+d+\gamma=$ $-1=\sum_{i=0}^{\infty}(p-1) p^{i}$, which is strictly periodic. Otherwise at least one coefficient is greater than 0 , so there is no borrow beyond this place when we add $c+d+\gamma$. Since $\gamma$ has period $L, \alpha+\beta$ has the same eventual period and its periodic part starts by index $k+L$. These are all the possibilities.

Now suppose $d>1$. We can write $\alpha=\sum_{i=0}^{\infty} a_{i} \pi^{i}=\sum_{j=0}^{d-1} \alpha_{j} \pi^{j}$ and $\beta=\sum_{i=0}^{\infty} a_{i} \pi^{i}=$ $\sum_{j=0}^{d-1} \beta_{j} \pi^{j}$ with $a_{i}, b_{i} \in\{0,1, \ldots, p-1\}$ and $\alpha_{j}, \beta_{j} \in \mathbf{Z}_{p}$. We have $\alpha_{j}=\sum_{i=0}^{\infty} a_{j, i} p^{j}$ and $\beta_{j}=\sum_{i=0}^{\infty} b_{j, i} p^{j}$ with $a_{j, i}=a_{d i+j}$ and $b_{j, i}=b_{d i+j}$. Let $M$ be the least common multiple of $d$ and $L$. Then

$$
a_{j, i+M / d}=a_{d i+M+j}=a_{d i+j}=a_{j, i} .
$$

That is, the coefficient sequence of $\alpha_{j}$ is periodic beyond the $\lceil(k-j) / d\rceil$ place with period $M / d$. Similarly for $\beta_{j}$. Thus (by the case $d=1$ ) the coefficient sequence $\alpha_{j}+\beta_{j}$ is periodic beyond index $\lceil(k-j) / d\rceil+M / d$ with period dividing $M / d$. The coefficient sequence of $\alpha+\beta$ is the interleaving of these $d$ sequences, (cf. Lemma 4.1) so it is periodic beyond index $k+M$ with period dividing $M$.

Finally, suppose that the coefficient sequences of $\alpha$ and $\beta$ are strictly periodic of period $L$ and that the period of $\alpha+\beta$ does not divide $L$. Let $\alpha+\beta=\sum_{i=0}^{\infty} e_{i} \pi^{i}$ with $0 \leq e_{i}<p$. Then for some $j$ we have $e_{j} \neq e_{j+L}$. But $e_{j}=a_{j}+b_{j}+c_{j}(\bmod p)$ and $e_{j+L}=a_{j+L}+b_{j+L}+c_{j+L}(\bmod p)=a_{j}+b_{j}+c_{j+L}$. Thus $a_{j}+b_{j}=p-1$ and we may assume that $c_{j}=1$ and $c_{j+L}=0$ (these values could be reversed, but then would need to be as assumed for some larger $j$ ). Let us take $j$ to be the least index such that $c_{j}=1$ and $c_{j+L}=0$.

Suppose that $j \geq d$. We must have $a_{j-d}+b_{j-d}+c_{j-d} \geq p$ and $a_{j+L-d}+b_{j+L-d}+$ $c_{j+L-d}<p$, and the only possibility is that $a_{j-d}+b_{j-d}=a_{j+L-d}+b_{j+L-d}=p-1$, $c_{j-d}=1$ and $c_{j+L-d}=0$, which contradicts the minimality of $j$. Therefore $j<d$. But this is impossible since there is no carry to any index $j<d$.

\section{Arithmetic Correlations}

In this section we consider only the binary case, $p=2$. Recall Klapper and Goresky [11] that a binary $\ell$ sequence is a periodic sequence of the form

$$
a_{i}=A 2^{-i}(\bmod N)(\bmod 2),
$$


where $N$ is a prime number such that 2 is a primitive root modulo $N$ (meaning that the powers of 2 account for all the non-zero elements of $\mathbf{Z} /(N)$ ). In this equation, $A \in \mathbf{Z} /(N)$, and $(\bmod N)(\bmod 2)$ means that first $A 2^{-i} \in \mathbf{Z} /(N)$ is represented as an integer between 0 and $N-1$, then this integer is reduced modulo 2 . The sequence has period $N-1$. Different choices of $A$ give different cyclic shifts of the same sequence.

Recall that Artin's conjecture states that for any integer $k \geq 2$, if $k$ is not a square, then there are infinitely many primes $N$ for which $k$ is a primitive root modulo $N$. (We are only interested in the case $k=2$ here.) Heilbronn conjectured, and Hooley [8] proved that if a certain extension of the Riemann hypothesis holds, then Artin's conjecture is true, and more precisely, $37.39558136 \%$ of all prime numbers $N$ have 2 as a primitive root. There has been considerable progress (Gupta and Murty [6] and Heath-Brown [7]) in removing the dependence on the Riemann hypothesis in these proofs. In any case, binary $\ell$-sequences are abundant.

As in Section 2 (specialized by setting $p=2$ ), let $\pi^{d}=2$, let $q \in \mathbf{Z}[\pi]$ and suppose that $(q)=\left(r^{t}\right)$ for some $r \in \mathbf{Z}[\pi]$ such that $n=N(r)$ is (an ordinary) prime number. (Then $N(q)=n^{t}$.) By Proposition 3.2, $\mathbf{Z}[\pi] /(q) \cong \mathbf{Z} /(N(q)) \cong \mathbf{Z} /\left(n^{t}\right)$. The group of invertible elements $\mathbf{Z}[\pi] /(q)^{*}$ in this ring is cyclic and has order $\phi\left(n^{t}\right)=n^{t-1}(n-1)$. In analogy with the simpler situation described in the preceding paragraph, we further assume that $\pi$ is a primitive element in $\mathbf{Z}[\pi] /(q)$, meaning that the $n^{t-1}(n-1)$ different powers of $\pi$ exactly account for the elements in $\mathbf{Z}[\pi] /(q)^{*}$. Fix $u \in \mathbf{Z}[\pi] /(q)^{*}$. We shall say that the resulting $d$-FCSR sequence,

$$
a_{i}=u \pi^{-i}(\bmod q)(\bmod \pi)
$$

is a generalized $\ell$-sequence with connection number $q$. The sequence is periodic of period $n^{t-1}(n-1)$. Different choices of $u$ give different cyclic shifts of the same sequence.

If $\mathbf{a}=a_{0}, a_{1}, \ldots$ is a binary sequence and $k \neq 0$ is an integer, then the sequence $\mathbf{b}=b_{0}, b_{1}, \ldots$ is said to be the $k$-fold decimation of $\mathbf{a}$ if $b_{i}=a_{k i}$ for every $i$. Let $\mathbf{a}$ be a generalized $\ell$-sequence. Let $\mathscr{F}_{\text {a }}$ be the family of all $k$-fold decimations of a, where $k \geq 1$ is allowed to vary over all integers which are relatively prime to the period of $\mathbf{a}$.

Let $\tau$ be a nonnegative integer. If $\mathbf{b}=b_{0}, b_{1}, \ldots$ is an infinite binary sequence, denote by $\mathbf{b}^{\tau}$ the shift of $\mathbf{b}$ by $\tau$ steps, that is, $b_{i}^{\tau}=b_{i+\tau}$. If $\beta=\sum_{i=0}^{\infty} b_{i} \pi^{i}$ is the $\pi$-adic integer corresponding to $\mathbf{b}$, let $\beta_{\tau}$ denote the $\pi$-adic integer corresponding to $\mathbf{b}^{\tau}$. That is,

$$
\beta_{\tau}=\frac{1}{\pi^{\tau}}\left(b-b_{0}-b_{1} \pi-\cdots-b_{\tau-1} \pi^{\tau-1}\right)=\sum_{i=0}^{\infty} b_{i+\tau} \pi^{i} .
$$

Recall that the ordinary cross-correlation with shift $\tau$ of two binary sequences $\mathbf{a}$ and b of period $L$ can be defined either as the sum $\sum_{i=1}^{N}(-1)^{s_{i}+t_{i+\tau}}$ or as the number of zeros minus the number of ones in one period of the bitwise exclusive - or of $\mathbf{a}$ and the $\tau$ shift of $\mathbf{b}$ [3]. The arithmetic cross-correlation is the with-carry analog of the latter definition. The following definition makes sense, because of Lemma 8.1. 
Definition 9.1. Let $\mathbf{a}$ and $\mathbf{b}$ be two periodic binary sequences with period $L$, with corresponding $\pi$-adic integers $\alpha, \beta \in \mathbf{Z}_{\pi}$. Let $0 \leq \tau<L$. The $d$-arithmetic crosscorrelation with shift $\tau, \Theta_{\mathbf{a}, \mathbf{b}}(\tau)$ of $\mathbf{a}$ and $\mathbf{b}$, is the number of zeros minus the number of ones in any periodic portion of length $L$ of the coefficient sequence for $\alpha-\beta_{\tau} \in \mathbf{Z}_{\pi}$. When $\mathbf{a}=\mathbf{b}$, the $d$-arithmetic cross-correlation is called the $d$-arithmetic autocorrelation of $\mathbf{a}$.

The sequences a, b (of period $L$ ) are said to have ideal $d$-arithmetic correlations if,

$$
\Theta_{\mathbf{a}, \mathbf{b}}(\tau)= \begin{cases}L & \text { if } \mathbf{a}=\mathbf{b}^{\tau} \\ 0 & \text { otherwise }\end{cases}
$$

for each $\tau$ with $0 \leq \tau<L$. A family of sequences is said to have ideal $d$-arithmetic correlations if every pair of sequences in the family has ideal $d$-arithmetic correlations. The second main result in this paper is the following.

THEOREM 9.2. Let $\pi^{d}=2$. Let $\mathbf{a}$ be a generalized $\ell$-sequence with connection integer $q \in \mathbf{Z}[\pi]$. Suppose that $(q)=\left(r^{t}\right)$ for some $r \in \mathbf{Z}[\pi]$ such that $N(r)$ is prime. Suppose also that $\operatorname{gcd}(d, \phi(N(q)))=1$. Then the family $\mathscr{F}_{\mathbf{a}}$ has ideal d-arithmetic correlations.

Remarks. The last two hypotheses are satisfied automatically if $N(q)$ is prime. One would like to find families with ideal $d$-arithmetic correlations such that whenever $\mathbf{a}, \mathbf{b}$ are distinct elements in the family then $\mathbf{a}$ and $\mathbf{b}^{\tau}$ are distinct (for all $\tau$ ), and such that whenever $\tau \neq 0$ then $\mathbf{a}$ and $\mathbf{a}^{\tau}$ are distinct. There is a certain amount of evidence that this happens quite often, cf. Klapper and Goresky [12].

The remainder of this section consists of a proof of Theorem 9.2. We first need a constraint on the sequences that can occur as generalized $\ell$-sequences.

PROPOSITION 9.3. Under the hypotheses of Theorem 9.2, the second half of one period of $\mathbf{a}$ is the bitwise complement of the first half.

Proof. Since $\mathbf{Z}[\pi] /(q) \cong \mathbf{Z} /(N(q))$, we have that

$$
\pi^{\phi(N(q))} \equiv 1(\bmod q)
$$

That is, $q$ divides

$$
\mid \pi^{n^{t-1}(n-1)}-1=\left(\pi^{n^{t-1}(n-1) / 2}-1\right)\left(\pi^{n^{t-1}(n-1) / 2}+1\right) .
$$

These two factors differ by 2 , so $r$ can only divide one of them. Thus $q$ divides exactly one of them. Since $\pi$ is primitive modulo $q, q$ cannot divide the first factor, hence it divides the second factor. It follows that $\pi^{\phi(N(q)) / 2} \equiv-1(\bmod q)$.

By Corollary 5.2 we have

$$
\begin{aligned}
a_{i+\phi(N(q)) / 2} & =\left(z m^{-i-\phi(N(q)) / 2}(\bmod N(q))\right)(\bmod 2) \\
& =\left(-z m^{-i}(\bmod N(q))\right)(\bmod 2),
\end{aligned}
$$


for some $z$. By Lemma 4.4, if $x$ is the element of $\Delta$ congruent to $-z m^{-i}$ modulo $q$, then the element of $\Delta$ that is congruent to $-z m^{-i}$ modulo $q$ is

$$
-q \frac{\pi^{d}-1}{\pi-1}-x \equiv 1-x(\bmod 2) .
$$

That is,

$$
a_{i+\phi(N(q)) / 2}=1-a_{i},
$$

which proves the proposition.

The above property is extended to decimations of generalized $\ell$-sequences by the following lemma.

LEMMA 9.4. Let a be a binary sequence of even period $L$ whose second half is the complement of its first half. Let $k>0$ be relatively prime to $L$, and let $\mathbf{b}$ be a $k$-fold decimation of $\mathbf{a}$. Then the second half of one period of $\mathbf{b}$ is the complement of the first half.

Proof. Note that $k$ must be odd and $a_{j}=1-a_{j+L / 2}$. Thus we have

$$
\begin{aligned}
b_{i} & =a_{i k} \\
& =1-a_{i k+L / 2} \\
& =1-a_{(i+L / 2) k} \\
& =1-b_{i+L / 2},
\end{aligned}
$$

which proves the proposition.

LEMMA 9.5. Let $L$ be an even integer. Let $\mathbf{b}=b_{0}, b_{1}, \ldots$ be a strictly periodic sequence with period $L$ such that the second half of each period is the bitwise complement of the first half and let

$$
\beta=\sum_{i=0}^{\infty} b_{i} \pi^{i}
$$

Then

$$
\beta=\frac{v}{\pi^{L / 2}+1},
$$

for some $v \in \mathbf{Z}[\pi]$.

Conversely, if $\mathbf{b}=b_{0}, b_{1}, \ldots$ is the $\pi$-adic expansion of a $\pi$-adic integer $w /\left(\pi^{L / 2}+1\right)$ with eventual period dividing $L, \operatorname{gcd}(L, d)=1$ and $w \notin\left\{0,\left(\pi^{L / 2}+1\right) /(\pi-1)\right\}$, then the second half of each $L$ bit period of $\mathbf{b}$ is the bitwise complement of the first half. 
Proof. If $\alpha$ is a $\pi$-adic number, then we let $\bar{\alpha}$ be the complementary $\pi$-adic number. That is, we replace each 1 by 0 and each 0 by 1 in the $\pi$-adic expansion of $\alpha$. For the first part, we have

$$
\begin{aligned}
\beta+\bar{\beta} & =1+\pi+\pi^{2}+\cdots \\
& =\frac{-1}{\pi-1} \\
& =-\left(1+\pi+\pi^{2}+\cdots+\pi^{d-1}\right),
\end{aligned}
$$

since $\pi^{d}=2$. Therefore

$$
\begin{aligned}
-\left(1+\pi+\pi^{2}+\cdots+\pi^{d-1}\right)-\beta & =\bar{\beta} \\
& =x+\pi^{L / 2} \beta,
\end{aligned}
$$

for some $x \in \mathbf{Z}[\pi]$. Solving for $\beta$ we have

$$
\beta=\frac{-x-1-\pi-\cdots-\pi^{d-1}}{\pi^{L / 2}+1},
$$

as claimed.

Now consider the converse. We first reduce to the case when $\mathbf{b}$ is strictly periodic. We can write

$$
\frac{w}{\pi^{L / 2}+1}=x+\pi^{k} \gamma,
$$

with $x \in \mathbf{Z}[\pi], k$ a positive integer, and $\gamma$ a $\pi$-adic integer with strictly periodic $\pi$-adic expansion. Since the $\pi$-adic expansion of $\gamma$ is periodic, we can write $\gamma=y /\left(\pi^{L}-1\right)$ for some $y \in \mathbf{Z}[\pi]$. Thus

$$
\left(w-x\left(\pi^{L / 2}+1\right)\right)\left(\pi^{L / 2}-1\right)=\pi^{k} y .
$$

In particular, $\pi^{k}$ divides the left hand side of equation (16). It follows that $\pi$ divides $w-x\left(\pi^{L / 2}+1\right)$, since $\pi^{L / 2}-1$ is congruent to -1 modulo $\pi$. Note that we must be careful here since $\mathbf{Z}[\pi]$ may not be a unique factorization domain. By induction, $\pi^{k}$ divides $w-x\left(\pi^{L / 2}+1\right)$. Thus we can write $w-x\left(\pi^{L / 2}+1\right)=\pi^{k} z$ for some $z \in \mathbf{Z}[\pi]$. Therefore

$$
\gamma=\frac{z}{\pi^{L / 2}+1},
$$

and we may assume $\mathbf{b}$ is strictly periodic.

Let

$$
\mu=\pi^{(d-1) L / 2}-\pi^{(d-2) L / 2}+\cdots-\pi^{L / 2}+1,
$$


so $\mu\left(\pi^{L / 2}+1\right)=\pi^{d L / 2}+1=2^{L / 2}+1$. Then

$$
\begin{aligned}
\frac{w}{\pi^{L / 2}+1} & =\frac{w \mu}{2^{L / 2}+1} \\
& =\frac{v_{0}}{2^{L / 2}+1}+\frac{v_{1}}{2^{L / 2}+1} \pi+\cdots+\frac{v_{d-1}}{2^{L / 2}+1} \pi^{d-1},
\end{aligned}
$$

for some rational integers $v_{0}, v_{1}, \ldots, v_{d-1}$. Since $\mathbf{b}$ is strictly periodic, we must have

$$
-\left(2^{L / 2}+1\right) \leq v_{j} \leq 0,
$$

for all $j$

We claim that if any of the $v_{j}$ is zero, then they all are, and that if any of the $v_{j}$ is $-\left(2^{L / 2}+1\right)$, then they all are. For any $j$ we have

$$
\frac{v_{j}}{2^{L / 2}+1}=\sum_{i=0}^{\infty} b_{d i+j} 2^{i} .
$$

Fix a particular $j$. Since $L$ and $d$ are relatively prime, we can choose $n, m \in \mathbf{Z}$ so that $n L+j=m d$, and we can assume that $n$ and $m$ are nonnegative. Then by the fact that b is periodic with period dividing $L$, for every $i$

$$
\begin{aligned}
b_{d i+j} & =b_{d i+n L+j} \\
& =b_{d(i+m)} .
\end{aligned}
$$

It follows that

$$
\begin{aligned}
\frac{v_{j}}{2^{L / 2}+1} & =\sum_{i=0}^{\infty} b_{d(i+m)} 2^{i} \\
& =2^{-d m} \sum_{i=m}^{\infty} b_{d i} 2^{i} \\
& =2^{-d m}\left(\frac{v_{0}}{2^{L / 2}+1}-x\right),
\end{aligned}
$$

where $x$ is a rational integer between 0 and $2^{m}-1$. That is, $2^{d m} v_{j}=v_{0}-\left(2^{L / 2}+1\right) x$. It follows that if $2^{L / 2}+1$ divides one of $v_{j}$ and $v_{0}$, then it divides the other. If $v_{0}=0$ and $v_{j}=-\left(2^{L / 2}+1\right)$, then $x=2^{d m}$, which is impossible. If $v_{0}=-\left(2^{L / 2}+1\right)$ and $v_{j}=0$, then $x=-1$, which is also impossible. The claim follows.

Now, by hypothesis the $v_{j}$ are not all zero and are not all $-\left(2^{L / 2}+1\right)$. Hence by the above claim, none is zero and none is $-\left(2^{L / 2}+1\right)$. In particular there is an exponential representation for $v_{0} /\left(2^{L / 2}+1\right), b_{d i}=\left(-v_{0} 2^{-i}\left(\bmod 2^{L / 2}+1\right)\right)(\bmod 2)$. 
Since $d$ is odd,

$$
\begin{aligned}
b_{d i+L / 2} & =b_{d(i+L / 2)} \\
& =\left(-v_{0} 2^{-i-L / 2}\left(\bmod 2^{L / 2}+1\right)\right)(\bmod 2) \\
& =\left(v_{0} 2^{-i}\left(\bmod 2^{L / 2}+1\right)\right)(\bmod 2) \\
& =1-b_{d i} .
\end{aligned}
$$

Now let $m$ be arbitrary and pick $i$ and $k$ so $m=i d+k L$ and $i \geq 0$. Then

$$
\begin{aligned}
b_{m+L / 2} & =b_{i d+k L+L / 2} \\
& =b_{i d+L / 2} \\
& =1-b_{i d} \\
& =1-b_{m-k L} \\
& =1-b_{m},
\end{aligned}
$$

which proves the lemma.

Theorem 9.2 is an immediate consequence of the following computation.

THEOREM 9.6. Let the hypotheses be as in Theorem 9.2. Let $k$ and $k^{\prime}$ be relatively prime to $\phi(N(q))$ with $1 \leq k, k^{\prime}<\phi(N(q))$. Let $\mathbf{b}$ and $\mathbf{c}$ be $k$ and $k^{\prime}$-fold decimations of a, respectively. Then the d-arithmetic cross-correlation of $\mathbf{c}$ and $\mathbf{d}$ with shift $\tau$ is

$$
\Theta_{\mathbf{c}, \mathbf{d}}(\tau)= \begin{cases}\phi(N(q)) & \text { if } \mathbf{c}=\mathbf{d}^{\tau} \\ 0 & \text { otherwise. }\end{cases}
$$

Proof. Let $L=\phi(N(q))$. Let $\mathbf{b}$ and $\mathbf{c}$ have associated $\pi$-adic numbers $\alpha^{\prime}=$ $a^{\prime} /\left(\pi^{L / 2}+1\right)$ and $\alpha^{\prime \prime}=a^{\prime \prime} /\left(\pi^{L / 2}+1\right)$, respectively. This is possible by Lemma 9.5 . The shift of $\mathbf{c}$ by $\tau$ corresponds to a $\pi$-adic integer $\pi^{L-\tau} \alpha^{\prime \prime}+x$ for some $x \in \mathbf{Z}[\pi]$. The $d$-arithmetic cross-correlation of $\mathbf{b}$ and $\mathbf{c}$ with shift $\tau$ is the number of zeros minus the number of ones in one length $L$ period of

$$
\begin{aligned}
\beta & =\alpha^{\prime}-\left(\pi^{L-\tau} \alpha^{\prime \prime}+x\right) \\
& =\frac{a^{\prime}-\pi^{L-\tau} a^{\prime \prime}-x\left(\pi^{L / 2}+1\right)}{\pi^{L / 2}+1} \\
& \stackrel{\text { def }}{=} \frac{b}{\pi^{L / 2}+1} .
\end{aligned}
$$

If $\mathbf{c}$ is a shift of $\mathbf{b}$ with shift $\tau$, then $\beta=0$ and the result follows.

Suppose $\mathbf{c}$ is not a shift of $\mathbf{b}$ with shift $\tau$. Let $\mathbf{d}$ be the sequence associated to $\beta$. It suffices to show that any period of $\mathbf{d}$ is balanced. The element $b$ is nonzero and by Lemma 8.1 the coefficient sequence of $b /\left(\pi^{L / 2}+1\right) \in \mathbf{Z}_{\pi}$ has eventual period dividing $L$. The theorem then follows from the second part of Lemma 9.5. 
Remark. It is not in general true that the coefficient sequence of a $\pi$-adic integer of the form $w /\left(\pi^{L / 2}+1\right)$ has eventual period dividing $L$. For example, if we take $w=-\pi^{L / 2}-1$, then the period is $d$. However, the $\pi$-adic integer $b /\left(\pi^{L / 2}+1\right)$ (which arises in the preceding proof) is the difference of two $\pi$-adic integers whose coefficient sequences have period dividing $L$.

\section{Acknowledgments}

Mark Goresky and Andrew Klapper would like to thank the Institue for Advanced Study in Princeton N.J. for its hospitality and support while this paper was being written. Research of Mark Goresky was partially supported by N.S.F. grant \# 0002693. Research of A. Klapper was partially supported by the National Science Foundation under grant \# 9980429.

\section{References}

1. L. Blum, M. Blum and M. Shub, A simple unpredictable pseudorandom number generator, SIAM J. Comp., Vol. 15 (1986) pp. 364-383.

2. Z. I. Borevich and I. R. Shefarevich, Number Theory, Academic Press, New York, N.Y. (1966).

3. S. Golomb, Shift Register Sequences, Aegean Park Press, Laguna Hills, CA (1982).

4. M. Goresky and A. Klapper, Feedback registers based on ramified extensions of the 2-adic numbers, Advances in Cryptology-Eurocrypt '94, Lecture Notes in Computer Science, Vol. 950, Springer Verlag, New York (1995).

5. M. Goresky and A. Klapper, Fibonacci and Galois representations for feedback with carry shift registers, IEEE Trans. Info. Theory, (2002) pp. 2826-2836.

6. R. Gupta and R. Murty, A remark on Artin's conjecture, Invent. Math., Vol. 78 (1984) pp. 127-130.

7. D. Heath-Brown, Artin's conjecture for primitive roots, Quart. J. Math. Oxford, Vol. 37 (1986) pp. 27-38.

8. C. Hooley, On Artin's conjecture, J. Reine Angew. Math., Vol. 22 (1967) pp. 209-220.

9. K. Ireland and M. Rosen, A Classical Introduction to Modern Number Theory, Springer-Verlag, New York (1990).

10. A. Klapper, Distribution properties of d-FCSR sequences, Journal of Complexity, Vol. 20 (2004) pp. 305-317.

11. A. Klapper and M. Goresky, Feedback shift registers, combiners with memory, and 2-adic span, Journal of Cryptology, Vol. 10 (1997) pp. 111-147.

12. A. Klapper and M. Goresky, Arithmetic cross-correlations of FCSR sequences, IEEE Trans. Info. Theory, Vol. 43 (1997) pp. 1342-1346.

13. A. Klapper and J. Xu, Algebraic feedback shift registers, Theoretical Computer Science, Vol. 226 (1999) pp. 61-93.

14. N. Koblitz, $p$-Adic Numbers, $p$-Adic Analysis, and Zeta Functions, Springer-Verlag, New York (1984).

15. S. Lang, Algebra, Addison-Wesley, Reading, MA (1965).

16. D. Mandelbaum, Arithmetic codes with large distance, IEEE Trans. Info. Theory, Vol. IT-13 (1967) pp. $237-242$

17. G. Marsaglia, The mathematics of random number generators, The Unreasonable Effectiveness of Number Theory, Amer. Math. Soc., Providence R. I. (1992) pp. 73-90.

18. G. Marsaglia and A. Zaman, A new class of random number generators, Annals of Applied Probability, Vol. 1 (1991) pp. 462-480.

19. J. Massey and R. Rueppel, Method of, and apparatus for, transforming a digital data sequence into an encoded form, U.S. Patent No. 4,797,922 (1989).

20. L. R. Welch, Lower bounds on the maximum correlation of signals, IEEE Trans. Info. Theory, Vol. IT-20 (1974) pp. 397-399. 